\title{
Quantum response theory for nonequilibrium steady states
}

\author{
Michael Konopik and Eric Lutz $\mathbb{}$ \\ Institute for Theoretical Physics I, University of Stuttgart, D-70550 Stuttgart, Germany
}

(Received 10 December 2018; published 6 December 2019)

\begin{abstract}
Quantum response theory is a cornerstone of statistical physics. However, the standard Kubo formalism is restricted to isolated equilibrium systems. We here generalize Kubo's results to open quantum systems in nonequilibrium steady states. We derive three different, but equivalent, forms of the quantum response function. We discuss for each of them the role of the noncommutativity of quantum operators and introduce a steady-state extension of the Kubo transformation. We show in particular that the equilibrium response vanishes for perturbations that commute with the unperturbed Hamiltonian, while the steady-state response does not, highlighting the profound difference between the two linear response theories.
\end{abstract}

DOI: 10.1103/PhysRevResearch.1.033156

\section{INTRODUCTION}

For equilibrium systems, the fluctuation-dissipation theorem connects the response to a weak external perturbation to the unperturbed correlation function between spontaneous fluctuations [1-3]. It offers a powerful tool to analyze general transport properties in numerous areas, from hydrodynamics to many-body and condensed-matter physics [4-8]. The fluctuation-dissipation relation has been derived for classical and closed quantum systems [4-8]. It is known to break down for nonequilibrium systems when detailed balance is not obeyed [9]. As a result, the determination of response functions for nonthermal systems has been a challenge for decades, despite their omnipresence in physical systems: energy, particle, and charge transport phenomena are prime examples.

Over the past years, the fluctuation-dissipation theorem has been successfully generalized to classical systems in nonequilibrium steady states, thus allowing the extension of response theory to this important class of nonthermal systems. Different theoretical formulations have been put forward [10-15], based on the Fokker-Planck equation [10], the overdamped Langevin dynamics [11-13], the Hatano-Sasa fluctuation theorem [14], or the dynamical activity [15]. Some of these modified fluctuation-response relations have been verified experimentally using colloidal particles in a toroidal optical trap [16-19].

Recently, Seifert and Speck have introduced a classification of steady-state fluctuation-dissipation theorems in the framework of stochastic thermodynamics, thus rationalizing previous methods that lead to apparently different results [20] (see also Ref. [21]). Using a classical master equation approach, they have identified three main equivalence classes:

Published by the American Physical Society under the terms of the Creative Commons Attribution 4.0 International license. Further distribution of this work must maintain attribution to the author(s) and the published article's title, journal citation, and DOI. the first variant contains a correlation function that involves no time derivatives (only functions of the steady-state distribution), the second variant is the unique form expressed in terms of time derivatives (of the stochastic entropy), whereas the last variant is the only one not requiring the explicit knowledge of the steady-state distribution. Infinitely many alternatives may be constructed via normalized linear combinations of the latter. All these variants yield the same response and are thus equivalent. However, the existence of different types of fluctuation-dissipation relations offers significant theoretical and experimental advantages. Theoretically, one kind of fluctuation-response theorem is usually easier to compute than the others, depending on the concrete application. At the same time, the choice of the form crucially affects the accuracy of the experimental determination of the nonequilibrium response function, as shown in Ref. [18]. Notwithstanding their central importance [22-25], a complete and unified picture of steady-state fluctuation-dissipation theorems for open quantum systems is currently missing.

We here develop a general framework for the steady-state response of open quantum systems, thus extending Kubo's theory which is limited to isolated equilibrium systems with unitary dynamics [3-8]. We specifically derive in Sec. II equivalence classes for generalized steady-state quantum fluctuation-dissipation relations using Markovian quantum Liouville equations and introduce a nonequilibrium extension of the Kubo transformation [3-8]. We discuss the role of the noncommutativity of quantum operators and emphasize differences to the equilibrium Kubo response theory, in particular the violation of detailed balance. To illustrate our unifying formalism, we analytically compute in Sec. III the response function to a step perturbation for an open system consisting of two weakly coupled harmonic oscillators, each interacting with a bath at a different temperature. The stationary state corresponds to an equilibrium state when the two temperatures are equal and to a nonequilibrium steady state when they are not. We use this model to stress the difference between classical and quantum responses, as well as between equilibrium and steady-state responses. Remarkably, we show that the equilibrium response function vanishes for perturbations 
that commute with the unperturbed Hamiltonian, whereas the steady-state response function does not. This underlines the profound disparity between equilibrium and nonequilibrium quantum response theories.

\section{QUANTUM EQUIVALENCE CLASSES}

We begin by deriving general equivalence classes for steady-state quantum response functions. We consider open quantum systems with Hamiltonians of the form $H(t)=H_{0}+$ $\varepsilon(t) H_{I}$, where $H_{0}$ is the unperturbed Hamiltonian and $\varepsilon(t) H_{I}$ the Hamiltonian perturbation with small time-dependent parameter $\varepsilon(t)$. We describe the open dynamics of the systems with density operator $\rho(t)$ using the Markovian quantum Liouville equation $d_{t} \rho(t)=\mathcal{L} \rho(t)$ [6]. We expand the Liouville superoperator $\mathcal{L}$ to first order in $\varepsilon(t)$ as $\mathcal{L}=\mathcal{L}_{0}+\varepsilon(t) \mathcal{L}_{1}+$ $O\left(\varepsilon^{2}\right)$. The dynamics is thus separated into an unperturbed part, $\mathcal{L}_{0} \cdot=-(i / \hbar)\left[H_{0}, \cdot\right]+\mathcal{D}[\cdot]$, where $\mathcal{D}$ is the dissipator induced by the nonunitary coupling to the bath [26], and a perturbed part $\varepsilon(t) \mathcal{L}_{1} \cdot=-(i \varepsilon(t) / \hbar)\left[H_{I}, \cdot\right]$. For fixed $\varepsilon$, we assume the existence of a stationary state $\pi_{\varepsilon}=\pi_{0}+\varepsilon \pi_{1}$ such that $\mathcal{L} \pi_{\varepsilon}=0$. Starting with the unperturbed steady state $\pi_{0}$, the linear response of the density operator $\rho(t)=\pi_{0}+\rho_{1}(t)$ to a time-dependent perturbation $\varepsilon(t)$ may be written as $\rho_{1}(t)=\int_{0}^{t} d s \varepsilon(s) e^{\mathcal{L}_{0}(t-s)} \mathcal{L}_{1} \pi_{0}$, where $e^{\mathcal{L}_{0}(t-s)}$ is the evolution superoperator of the unperturbed system $H_{0}$. The response of any observable of interest $A$ may then be calculated to linear order as

$$
\begin{aligned}
\langle A\rangle_{\varepsilon}(t) & =\langle A\rangle+\int_{0}^{t} d s \varepsilon(s) \operatorname{Tr}\left\{A e^{\mathcal{L}_{0}(t-s)} \mathcal{L}_{1} \pi_{0}\right\} \\
& =\langle A\rangle+\int_{0}^{t} d s \varepsilon(s) \mathcal{R}(t-s) .
\end{aligned}
$$

Here $\langle A\rangle_{\varepsilon}(t)=\operatorname{Tr}\{A \rho(t)\}$ denotes the perturbed expectation value of $A$ and $\langle A\rangle=\operatorname{Tr}\left\{A \pi_{0}\right\}$ the corresponding unperturbed expectation value. The response function is given by $\mathcal{R}(\tau)=$ $\operatorname{Tr}\left\{A e^{\mathcal{L}_{0} \tau} \mathcal{L}_{1} \pi_{0}\right\}$ with $\tau=t-s$. For equilibrium states $\pi_{0}$, Eq. (1) leads to the usual Kubo response theory for closed quantum systems for which $\mathcal{L}_{0} \cdot=-(i / \hbar)\left[H_{0}, \cdot\right][6,7]$. For nonequilibrium steady states $\pi_{0}$, it provides the basis for our quantum extension of the three equivalence classes identified in Ref. [20]. Despite their formal similarity, the two cases are fundamentally different: while the equilibrium response satisfies detailed balance [27], the steady-state response does not.

\section{A. Class one}

The first form $\mathcal{R}_{1}(\tau)$ of the quantum response function is expressed as a correlation function with an observable $B_{1}=$ $\left(\mathcal{L}_{1} \pi_{0}\right) / \pi_{0}$. It follows from Eq. (1) by introducing the adjoint time evolution of the unperturbed dynamics $A(\tau)=A e^{\mathcal{L}_{0} \tau}$ [26] and reads

$$
\mathcal{R}_{1}(\tau)=\left\langle A(\tau) B_{1}\right\rangle=\left\langle A(\tau)\left(\mathcal{L}_{1} \pi_{0}\right) / \pi_{0}\right\rangle .
$$

Expression (2) is a quantum generalization of the response function derived in Ref. [10] and is often referred to as the Agarwal form for this reason [20,21]. This form readily shows that for a thermal stationary distribution, $\pi_{0}=$ $\exp \left(-\beta H_{0}\right) / Z_{0}$, with $Z_{0}$ the partition function, the quantum response vanishes when the perturbation commutes with the unperturbed Hamiltonian, $\left[H_{I}, H_{0}\right]=0$. This is not necessarily the case for a quantum nonequilibrium steady state, as we will discuss in detail below. This variant of the fluctuationresponse theorem is distinguished by the fact that it contains only state variables and no time derivatives. Its drawback is that the observable $B_{1}$ involves the stationary distribution $\pi_{0}$, which is not always explicitly known in concrete situations.

\section{B. Class two}

In the classical regime, the second variant is written in terms of the time derivative of the $\varepsilon$ derivative of the stochastic entropy of the system, $\left.\partial_{\varepsilon} S_{\varepsilon}\right|_{0}=-\left.\partial_{\varepsilon} \ln \pi_{\varepsilon}\right|_{0}=$ $-\pi_{1} / \pi_{0}$, along single trajectories $[10,20]$. We obtain the second form $\mathcal{R}_{2}(\tau)$ of the quantum response function by noting that the stationary state of the Liouvillian has to vanish at every order, $\left(\mathcal{L}_{0}+\varepsilon \mathcal{L}_{1}\right)\left(\pi_{0}+\varepsilon \pi_{1}\right)=\mathcal{L}_{0} \pi_{0}+$ $\varepsilon\left(\mathcal{L}_{1} \pi_{0}+\mathcal{L}_{0} \pi_{1}\right)+O\left(\varepsilon^{2}\right)=0$. As a result, $\mathcal{L}_{0} \pi_{0}=0$ and $\mathcal{L}_{1} \pi_{0}=-\mathcal{L}_{0} \pi_{1}$. Since $\mathcal{L}_{0}$ is the generator of the open dynamics, we have $e^{\mathcal{L}_{0}(t-s)} \mathcal{L}_{0} \rho=d_{t} e^{\mathcal{L}_{0}(t-s)} \rho=d_{\tau} e^{\mathcal{L}_{0} \tau} \rho=$ $-d_{s} e^{\mathcal{L}_{0}(t-s)} \rho$. The response function in Eq. (1) may therefore be rewritten as

$$
\mathcal{R}_{2}(\tau)=-\operatorname{Tr}\left\{A(\tau) \mathcal{L}_{0} \pi_{1}\right\}=-d_{\tau}\left\langle A(\tau) \pi_{1} / \pi_{0}\right\rangle .
$$

In the limit of closed quantum systems at equilibrium, Eq. (3) reduces to the Kubo quantum response function, $\mathcal{R}_{\mathrm{K} 1}(\tau)=$ $-\beta d_{\tau}\left\langle A(\tau) \tilde{H}_{I}\right\rangle$, since $\pi_{1} / \pi_{0}=\beta \tilde{H}_{I}=\int_{0}^{\beta} d \lambda e^{-\lambda H_{0}} H_{I} e^{\lambda H_{0}}$ is the Kubo transform of $H_{I}$ [3]. The advantage of the Kubo transformation is that it allows us to formulate classical and quantum equilibrium response functions in the same form by simply replacing an operator by its corresponding transform. Such a procedure can be carried over to steady-state response functions. In order to bring Eq. (3) in a form similar to the classical case, we first introduce a generalized Kubo transformation: Using the identity $\partial_{\varepsilon} \pi_{\varepsilon}=\int_{0}^{1} \pi_{\varepsilon}^{\lambda}\left(\partial_{\varepsilon} \ln \pi_{\varepsilon}\right) \pi_{\varepsilon}^{1-\lambda} d \lambda$, which holds for any density operator [28], we obtain, for $\varepsilon=$ $0,\left.\partial_{\varepsilon} \pi_{\varepsilon}\right|_{0} / \pi_{0}=\left.\int_{0}^{1} \pi_{0}^{\lambda}\left(\partial_{\varepsilon} \ln \pi_{\varepsilon}\right)\right|_{0} \pi_{0}^{-\lambda} d \lambda=: \overline{\left.\partial_{\varepsilon} \ln \pi_{\varepsilon}\right|_{0}}$, where we have defined the transform $\overline{\left.\partial_{\varepsilon} \ln \pi_{\varepsilon}\right|_{0}}$ of $\left.\partial_{\varepsilon} \ln \pi_{\varepsilon}\right|_{0}$. The latter reduces to the usual Kubo transform for a thermal state, $\pi_{\varepsilon}=e^{-\beta\left(H_{0}+\varepsilon H_{I}\right)} / Z_{\varepsilon}$. It may thus be regarded as a steady-state extension of the Kubo-Mori-Bogoliubov inner product [29]. We accordingly find

$$
\mathcal{R}_{2}(\tau)=-d_{\tau}\left\langle A(\tau) \overline{\left.\partial_{\varepsilon} \ln \pi_{\varepsilon}\right|_{0}}\right\rangle=d_{\tau}\left\langle A(\tau) \overline{\left.\partial_{\varepsilon} S_{\varepsilon}\right|_{0}}\right\rangle,
$$

where we have introduced the quantum analog of the stochastic entropy $S_{\varepsilon}=-\ln \pi_{\varepsilon}$. Noting furthermore that two-time correlation functions for open quantum systems are defined as $\langle A(t) B(s)\rangle=\operatorname{Tr}\left\{A e^{\mathcal{L}_{0}(t-s)} B e^{\mathcal{L}_{0} s} \rho(0)\right\}[26,30]$, we obtain, with $\rho(0)=\pi_{0}$ and $d_{s} e^{\mathcal{L}_{0} s} \pi_{0}=0$,

$$
\mathcal{R}_{2}(\tau)=-d_{s}\left\langle A(\tau) \overline{\left.\partial_{\varepsilon} S_{\varepsilon}\right|_{0}}\right\rangle=-\left\langle A(t) d_{s} \overline{\left.\partial_{\varepsilon} S_{\varepsilon}(s)\right|_{0}}\right\rangle .
$$

Formula (5) is a quantum extension of the response function of Refs. [10,20]. It can be formally written in Liouville space as a correlation function with the observable $B_{2}(s)=-d_{s} \overline{\left.\partial_{\varepsilon} S_{\varepsilon}(s)\right|_{0}}$. In general, for noncommuting operators, $\left.\partial_{\varepsilon} \ln \left(\pi_{0}+\varepsilon \pi_{1}\right)\right|_{0} \neq \pi_{1} / \pi_{0}$, implying that $\overline{\partial_{\varepsilon} S_{\varepsilon}} \neq \partial_{\varepsilon} S_{\varepsilon}$. Consequently, the quantum response function (5) cannot be written in terms of the stochastic entropy, $-\left\langle\left. A(t) d_{s} \partial_{\varepsilon} S_{\varepsilon}(s)\right|_{0}\right\rangle$, as in the classical limit, unless $\left[\pi_{0}, \pi_{1}\right]=0$ (Appendix A). 
The variant (5) is the only one where the response function is given as a correlation function with a time derivative of a state variable, namely the formal time derivative of the generalized Kubo transformed $\varepsilon$ derivative of the stochastic entropy, $\overline{\left.\partial_{\varepsilon} S_{\varepsilon}\right|_{0}}$. The results presented in Refs. [23,24] are related to this variant (Appendix B).

We additionally mention that there is an alternative way of writing the quantum response function (5) without using any correlation function. We indeed have

$$
\mathcal{R}_{2, \text { alt }}(\tau)=-\left.d_{\tau} \partial_{\varepsilon} \operatorname{Tr}\left\{A(\tau) \pi_{\epsilon}\right\}\right|_{0}=-\left.d_{\tau} \partial_{\varepsilon}\langle A(\tau)\rangle_{\varepsilon}\right|_{0},
$$

where $\langle A(\tau)\rangle_{\varepsilon}=\operatorname{Tr}\left\{A(\tau) \pi_{\varepsilon}\right\} \neq\langle A\rangle_{\varepsilon}(\tau)$ is the perturbed expectation value of the observable $A(\tau)=A e^{\mathcal{L}_{0} \tau}$ evolved via the unperturbed dynamics. This form offers an intuitive interpretation of dynamic response theory: At any fixed time, $\left.\partial_{\varepsilon}\langle A(\tau)\rangle_{\varepsilon}\right|_{\varepsilon=0}$ can be seen as the static susceptibility, that is, the static response of the system to the external perturbation [6]. The dynamic response function (6) then follows as the time derivative of the time-dependent susceptibility. This form often enables a simple evaluation of the response function (see below).

\section{Class three}

Classically, the third form is the unique one that does not explicitly involve the stationary distribution $[15,20]$. This type of fluctuation-response relation is therefore of advantage when the steady-state distribution is not specifically known. Within our quantum Hamiltonian perturbation approach, such a variant may be derived from Eq. (2) by realizing that $\mathcal{L}_{1} \cdot=-(i / \hbar)\left[H_{I}, \cdot\right]$. We then obtain

$$
\mathcal{R}_{3}(\tau)=\frac{i}{\hbar} \operatorname{Tr}\left\{\pi_{0}\left[H_{I}, A(\tau)\right]\right\}=-\left\langle\mathcal{L}_{1} A(\tau)\right\rangle .
$$

In contrast to Eqs. (2)-(6), the response function (7) is given as an expectation value of operators that do not explicitly depend on either $\pi_{0}, \pi_{1}$, or $\pi_{\varepsilon}$ (see also Ref. [22] for an alternative approach). In the limit of unitary quantum systems at equilibrium, Eq. (7) reduces to the Kubo response function $\mathcal{R}_{\mathrm{K} 2}(\tau)=(i / \hbar)\left\langle\left[H_{I}, A(\tau)\right]\right\rangle$ [3]. Interestingly, expression (7) indicates that the quantum response function vanishes when the time evolved observable $A(\tau)$ commutes with the perturbation Hamiltonian $H_{I}$.

\section{Detailed balance}

Equilibrium response functions obey the detailed balance condition $\langle A B(t)\rangle=\langle B A(t)\rangle$ [27]. By contrast, the steadystate response functions that we have here derived do not, as we show explicitly for the example below. In particular, while the steady-state response functions (1) and (7) formally look like their equilibrium counterparts, they do violate detailed balance, that is, $\left\langle A\left[\left(\mathcal{L}_{1} \pi_{0}\right) / \pi_{0}\right](\tau)\right\rangle \neq\left\langle\left(\mathcal{L}_{1} \pi_{0}\right) / \pi_{0} A(\tau)\right\rangle$ and $\left\langle\left[H_{I}, A(\tau)\right]\right\rangle \neq\left\langle\left[A, H_{I}(\tau)\right]\right\rangle$ for steady states. This has profound physical consequences as we have discussed above: The (detailed-balance-obeying) equilibrium response functions indeed vanish for perturbations that commute with the unperturbed Hamiltonian, whereas (detailed-balance-violating) steady-state response functions do not.

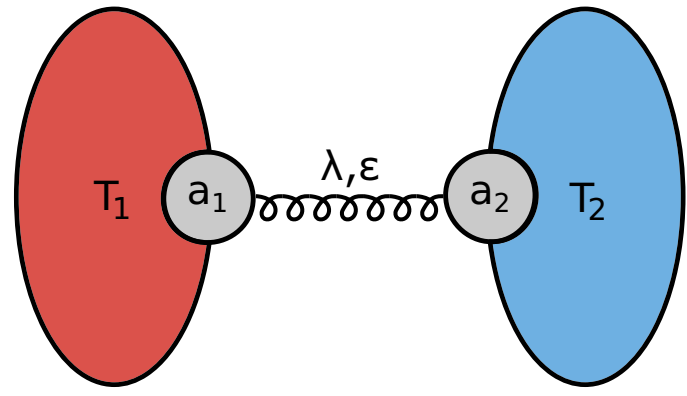

FIG. 1. Steady-state model. Two quantum harmonic oscillators are weakly coupled to each other with coupling strength $\lambda$. Each of them interacts with a bath with a different temperature $T_{j}$, $(j=1,2)$. A nonequilibrium steady state is established when the two temperatures are different and heat flows from one oscillator to the other. We examine the response of oscillator 1 when the coupling is modulated by $\varepsilon(t)$

\section{EXAMPLE}

Our results are applicable to general open quantum systems. As an illustration, we now consider a system consisting of two weakly coupled harmonic oscillators, each interacting with its own reservoir at a different temperature (Fig. 1). This model describes the coupling of a radiation mode with a vibrational mode in cavity optomechanics [31], of two Bose condensates in bosonic Josephson junctions [32], as well as the weak coupling limit of the Dicke Hamiltonian [33]. By properly tuning the parameters of the system, this model allows one to compare different response regimes: unitary/dissipative, equilibrium/steady state, and classical/quantum. In particular, a nonequilibrium steady state is established when the two bath temperatures are different. The (unperturbed) Hamiltonian of the system is [31-33]

$$
H_{0}=\hbar \omega_{1} a_{1}^{\dagger} a_{1}+\hbar\left(\omega_{1}+\delta\right) a_{2}^{\dagger} a_{2}+\hbar \lambda\left(a_{1} a_{2}^{\dagger}+a_{1}^{\dagger} a_{2}\right),
$$

where $\left(a_{j}^{\dagger}, a_{j}\right)(j=1,2)$ are the ladder operators of the oscillators with respective frequencies $\omega_{1}$ and $\omega_{2}=\omega_{1}+\delta$ (with detuning $\delta$ ). The coupling parameter is denoted by $\lambda$. The (unperturbed) Liouville superoperator reads [34]

$$
\mathcal{L}_{0} \cdot=-(i / \hbar)\left[H_{0}, \cdot\right]+\sum_{j=1}^{2} \mathcal{D}_{j}[\cdot],
$$

with the two nonunitary dissipators $\mathcal{D}_{j}$ induced by the interaction with the heat reservoirs,

$$
\begin{aligned}
\mathcal{D}_{j}[\rho]= & \gamma\left(n_{j}+1\right)\left[a_{j} \rho a_{j}^{\dagger}-\frac{1}{2}\left(a_{j}^{\dagger} a_{j} \rho+\rho a_{j}^{\dagger} a_{j}\right)\right] \\
& +\gamma n_{j}\left[a_{j}^{\dagger} \rho a_{j}+\frac{1}{2}\left(a_{j} a_{j}^{\dagger} \rho+\rho a_{j} a_{j}^{\dagger}\right)\right] .
\end{aligned}
$$

Here $n_{j}=\left[\exp \left(\beta_{j} \omega_{j}\right)-1\right]^{-1}$ is the thermal occupation number at inverse temperature $\beta_{j}$ and $\gamma$ is the damping constant. For concreteness, we apply a step perturbation $\varepsilon(t) H_{I}=$ $\hbar \varepsilon(t)\left(a_{1} a_{2}^{\dagger}+a_{1}^{\dagger} a_{2}\right)$, with $\varepsilon(t)=\varepsilon \Theta(t)$, to the coupling between the harmonic oscillators and look at the response of the (dimensionless) energy of the first oscillator, $A=\beta_{1} \hbar \omega_{1} a_{1}^{\dagger} a_{1}$. We note that the unperturbed system is in a thermal state for $\lambda=0$ and in a nonequilibrium steady state for $\lambda \neq 0$. The 


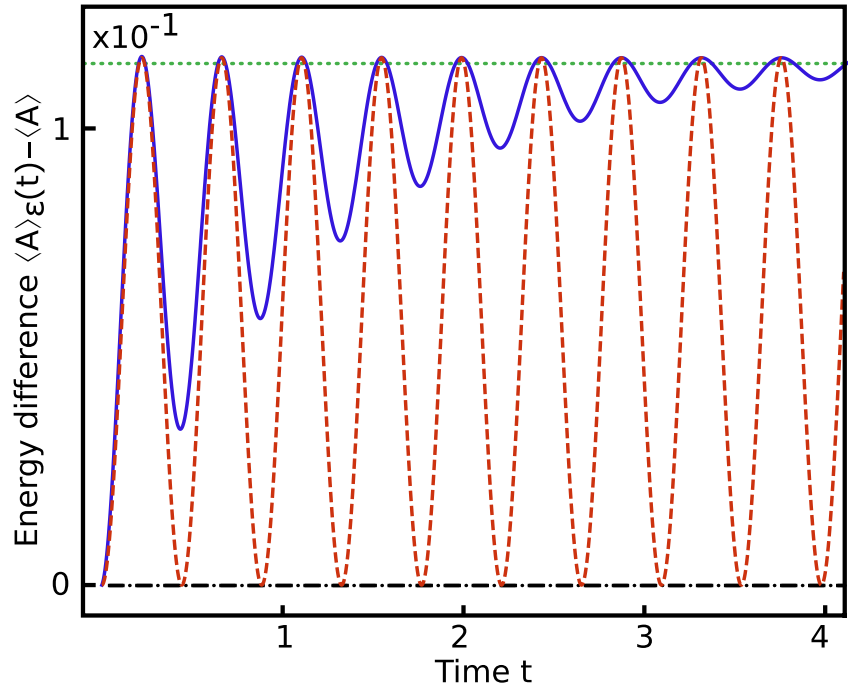

FIG. 2. Quantum response of the (dimensionless) energy of the first oscillator, $\langle A\rangle_{\varepsilon}(t)-\langle A\rangle$, with $A=\beta_{1} \hbar \omega_{1} a_{1}^{\dagger} a_{1}$, to a step perturbation, $\varepsilon(t)=\varepsilon \Theta(t)$, of the coupling between the two harmonic oscillators. The steady-state response $(\lambda \neq 0)$ (blue solid), Eq. (11), asymptotically approaches the perturbed value (green dotted). By contrast, the equilibrium response $(\lambda=0)$ (black dotted-dashed) vanishes and the unitary response $(\gamma=0)$ (red dashed), Eq. (12), keeps oscillating and fails to reach the perturbed value of the observable $A$. Parameters are $\omega_{1}=2.4, \delta=10.1, \gamma=0.7, \lambda=5$, $\varepsilon=0.11, \beta_{1}=0.092$, and $\beta_{2}=0.0008$.

two quantum oscillators are moreover closed with unitary dynamics in the absence of damping, $\gamma=0$. Finally, the classical regime is achieved in the high-temperature limit $\beta_{j} \hbar \omega_{j} \ll 1$.

We determine the quantum response function using the forms (6) and (7) (Appendix C). To this end, we first evaluate the steady-state density operator of the system and then calculate the time dependence of observable $A$. Due to the quadratic nature of the Hamiltonian, it is convenient to employ the Gaussian characteristic function $\chi\left(x_{1}, p_{1}, x_{2}, p_{2}\right)=$ $\exp \left(i \vec{P} \overrightarrow{\bar{y}}-\vec{P}^{T} \bar{\sigma} \vec{P} / 2\right)$ of the system, with coordinate vector $\vec{P}=\left(x_{1}, p_{1}, x_{2}, p_{2}\right)^{T}$, symplectic mean vector $\overrightarrow{\bar{y}}$, and covariance matrix $\bar{\sigma}$ to compute the stationary distribution [34]. The time evolution of operator $A$ is obtained via matrix exponentiation. We find

$$
\begin{aligned}
\mathcal{R}_{3}(\tau) & =i \beta_{1} \hbar \omega_{1}\left\langle\left[a_{1} a_{2}^{\dagger}+a_{1}^{\dagger} a_{2}, a_{1}^{\dagger} a_{1}(\tau)\right]\right\rangle \\
& =e^{-\gamma \tau} \frac{\gamma\left(\delta^{2}+4 \lambda^{2} \cos z \tau\right)+\left(\gamma^{2}+\delta^{2}\right) z \sin z \tau}{z^{2}\left(\gamma^{2}+z^{2}\right)\left(2 \lambda \Delta n \beta_{1} \hbar \omega_{1}\right)^{-1}} \\
& =-\left.\beta_{1} \hbar \omega_{1} d_{\tau} \partial_{\varepsilon}\left\langle a^{\dagger} a(\tau)\right\rangle\right|_{\varepsilon=0}=\mathcal{R}_{2, \text { alt }}(\tau),
\end{aligned}
$$

where $\Delta n=n_{2}-n_{1}$ and $z=\sqrt{\delta^{2}+4 \lambda^{2}}$. The two forms $\mathcal{R}_{2 \text {,alt }}(\tau)$ and $\mathcal{R}_{3}(\tau)$ thus yield the same result, as expected. However, this is not obvious from their definitions (6) and (7), since $\mathcal{R}_{2 \text {,alt }}(\tau)$ displays a $\varepsilon$ dependence, while $\mathcal{R}_{3}(\tau)$ does not. The response function (11) does not obey detailed balance, $\mathcal{R}_{3}(\tau)=i\left\langle\left[H_{I}, a_{1}^{\dagger} a_{1}(\tau)\right]\right\rangle \neq$ $i\left\langle\left[a_{1}^{\dagger} a_{1}, H_{I}(\tau)\right]\right\rangle=\overline{\mathcal{R}}_{3}(\tau)$ (see Fig. 3 inset and Appendix D).

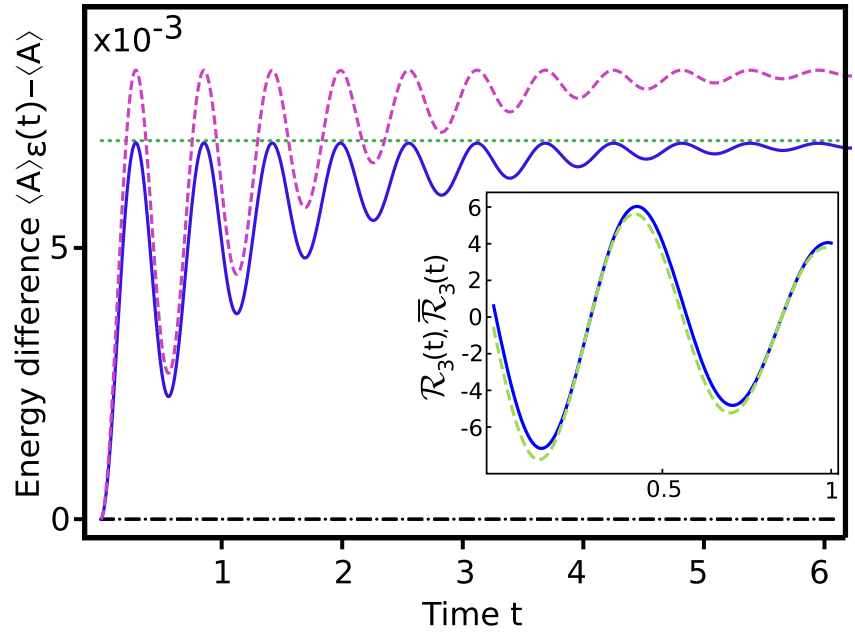

FIG. 3. Steady-state response of the (dimensionless) energy of the first oscillator, $\langle A\rangle_{\varepsilon}(t)-\langle A\rangle$, with $A=\beta_{1} \hbar \omega_{1} a_{1}^{\dagger} a_{1}$, to a step perturbation, $\varepsilon(t)=\varepsilon \Theta(t)$, of the coupling between the two harmonic oscillators. The quantum response $\left(\beta_{1} \hbar \omega_{1} \gg 1\right)$ (blue solid), Eq. (11), asymptotically approaches the perturbed value (green dotted). By contrast, the classical response $\left(\beta_{1} \hbar \omega_{1} \ll 1\right)$ (purple dashed), Eq. (13), although proportional to the quantum response fails to reach the perturbed value of the observable $A$. Inset: detailed balance is violated, $\mathcal{R}_{3}(t)=i\left\langle\left[H_{I}, a_{1}^{\dagger} a_{1}(t)\right]\right\rangle \neq i\left\langle\left[a_{1}^{\dagger} a_{1}, H_{I}(t)\right]\right\rangle=\overline{\mathcal{R}}_{3}(t)$. Parameters are $\omega_{1}=2.4, \delta=10.1, \gamma=0.7, \lambda=2.3, \varepsilon=0.11$, $\beta_{1}=0.164$, and $\beta_{2}=0.416$.

Three different response regimes may be distinguished, as can be seen in Figs. 2 and 3, that represent the response difference $\langle A\rangle_{\varepsilon}(t)-\langle A\rangle$ for various system parameters:

(i) In the thermal limit $\lambda \rightarrow 0$, the unperturbed quantum oscillator is in an equilibrium state and the quantum response function (11) vanishes (black dotted-dashed line). By contrast, the steady-state response is different from zero (blue solid line) and approaches the perturbed stationary value at large times (green dotted line). This example emphasizes the fundamental difference between equilibrium and steadystate quantum response theories. The response function can actually be used to distinguish thermal and nonthermal states via their different behavior for a perturbation that commutes with $H_{0}$ for $\delta=0$ (Appendix E).

(ii) In the unitary limit $\gamma=0$, when the interaction with the two heat reservoirs is switched off, the quantum response function (11) reduces to

$$
\mathcal{R}_{\text {unitary }}(\tau)=2 \lambda \Delta n \beta_{1} \hbar \omega_{1} \frac{\delta^{2}}{z^{3}} \sin z \tau .
$$

We observe (Fig. 2) that the perturbed observable (red dashed line) exhibits in this situation oscillations with the same oscillation period as in the nonunitary case $(\gamma \neq 0)$ (blue solid line). However, it never reaches its perturbed value (green dotted line) due to the absence of external damping.

(iii) Finally, in the classical limit, $\beta_{j} \hbar \omega_{j} \ll 1$, the Bose distribution reduces to the Boltzmann distribution and the response function (11) simplifies to

$$
\mathcal{R}_{\text {classical }}(\tau)=\frac{\beta_{1} \omega_{1}-\beta_{2} \omega_{2}}{\beta_{2} \omega_{2}} \frac{\mathcal{R}_{3}(\tau)}{\Delta n \beta_{1} \hbar \omega_{1}} .
$$


The classical response function (13) is hence proportional to the quantum response function (11). However, it predicts the wrong perturbed value of the observable $A$ (purple dotted line in Fig. 3), stressing the difference between classical and quantum response theories.

\section{CONCLUSIONS}

We have generalized Kubo's response theory to nonequilibrium open quantum systems described by Markovian Liouville equations. We have, in particular, introduced a steady-state extension of the Kubo transformation. We have concretely derived quantum generalizations of the equivalence classes for classical response functions introduced in Ref. [20]. We have for each of them analyzed the role of noncommuting operators and identified conditions under which the quantum response vanishes when some operators commute. We have further illustrated our results with an analytically solvable model of two weakly coupled open quantum harmonic oscillators and compared various response regimes including unitary/dissipative, equilibrium/steady state, and classical/quantum limits. We have shown, in addition, that the equilibrium response can vanish in instances where the steady-state response does not, since detailed balance is violated. Our findings not only provide a unified picture of nonequilibrium quantum response theory, they also offer different, but equivalent, approaches to evaluate steady-state response functions, depending on the specific problem considered. We thus expect them to be useful for future studies of the quantum response of nonequilibrium steady-state systems.

\section{ACKNOWLEDGMENT}

We thank Tobias Donner for discussions and acknowledge support from the DFG (Contract No. FOR 2724).

\section{APPENDIX A: PROPERTIES OF THE SECOND FORM}

We here show that the response function $R_{2}(\tau)$ vanishes for a thermal state when $\left[\pi_{0}, \pi_{1}\right]=0$. For commuting distributions, we indeed have $\left.\partial_{\varepsilon} \ln \left(\pi_{0}+\varepsilon \pi_{1}\right)\right|_{0}=\pi_{1} / \pi_{0}$. Moreover, for a thermal state $\pi_{\varepsilon} \propto e^{-\beta\left(H_{0}+\varepsilon H_{I}\right)}$, the operator $\pi_{1}$ can be given explicitly via the Kubo transform, $\pi_{1} \propto \tilde{H}_{I} \pi_{0}$ [see Eq. (7.12) in Ref. [6]]. As a result, the condition $\left[\pi_{0}, \pi_{1}\right]=0$ implies that $\left[H_{0}, H_{I}\right]=0$ since the Kubo transform $\beta \tilde{H}_{I}=$ $\int_{0}^{\beta} d \lambda e^{-\lambda H_{0}} H_{I} e^{\lambda H_{0}}$ consists of exponentials that are proportional to $H_{0}$. Equation (2) of the main text then shows that $\mathcal{L}_{1} \pi_{0}=0$ and that the response function thus vanishes.

In the unitary limit, the formal identity in Liouville space for the correlation function $\left\langle A(t) d_{s} \overline{\left.\partial_{\varepsilon} S_{\varepsilon}(s)\right|_{0}}\right\rangle$ becomes one at the operator level in Hilbert space. In that case, the unitary time-evolution operators $A e^{\mathcal{L}_{0} t}=$ $U^{\dagger}(t, 0) A U(t, 0)=A(t)$ can be used to obtain the Heisenberg representation of the generalized stochastic entropy $\left\{U^{\dagger}(t, s) A U(t, s) B U(s, 0) \rho(0) U^{\dagger}(s, 0)\right\}=\langle A(t) B(s)\rangle$. The latter equality gives the observable $-d_{s} \overline{\left.\partial_{\varepsilon} S_{\varepsilon}(s)\right|_{0}}$ a meaning in Hilbert space.

\section{APPENDIX B: COMPARISON WITH OTHER APPROACHES}

Quantum steady-states response functions have been obtained in Refs. [23,24] using different methods. We here show that these results are related to the second class given by Eq. (3).

Chetrite and Mallick have derived a steady-state fluctuation-dissipation relation from a quantum JarzynskiHatano-Sasa fluctuation theorem for the instantaneous density operator $\pi_{t}$ for a frozen time $t$ [23]. This accompanying density operator satisfies $\mathcal{L}(t) \pi_{t}=0$ for each time $t$, but depends explicitly on time, $d_{t} \pi_{t} \neq 0$. It is therefore different from the true density operator $\rho_{t}$. Considering a modified superoperator (using the notation of this paper) $\mathcal{L}^{\prime}(t)=\mathcal{L}(t)+d_{t} \pi_{t} / \pi_{t}=: \mathcal{L}(t)+W_{t}$ they have derived a quantum Jarzynski-Hatano-Sasa relation of the form

$$
\operatorname{Tr}\left\{\pi_{t} A\right\}=\left\langle\overrightarrow{\exp }\left(\int_{0}^{t} W_{u} d u\right) A(t)\right\rangle_{0},
$$

where $\overrightarrow{\exp }$ is the time-ordered exponential. Taking the functional derivative of Eq. (B1) with $\mathcal{L}(t)=\mathcal{L}_{0}+\varepsilon(t) \mathcal{L}_{1}$ yields,

$$
\mathcal{R}_{\text {Chetrite }}(t)=-\frac{d}{d t}\left\langle A(t) \pi_{1} \pi_{0}^{-1}\right\rangle,
$$

which is equivalent to Eq. (3).

On the other hand, Mehboudi, Sanpera, and Parrondo have derived a steady-state response function for open quantum systems described by Markovian completely positive and trace-preserving maps $\zeta_{\varepsilon}(\rho)$ in terms of the symmetric logarithmic derivative (SLD) [24]. Assuming that each of these maps has an invariant state $\zeta_{\varepsilon} \pi_{\varepsilon}$, they have considered these maps and their invariant states up to linear order, $\zeta_{\varepsilon}=\zeta_{0}+\varepsilon \zeta_{1}$ and $\pi_{\varepsilon}=\pi_{0}+\varepsilon \pi_{1}$. For discrete time steps, the time evolution is given by $\rho(t)=\zeta_{\varepsilon(t)} \circ \zeta_{\varepsilon(t-1)} \circ \cdots \circ \zeta_{\varepsilon(1)} \pi_{0}$. Expanding this evolution equation up to linear order of $\varepsilon$ and taking the continuous limit, they arrived at

$$
\mathcal{R}_{\text {Mehboudi }}(t)=-d_{t}\left\langle A(t) \Lambda_{0}+\Lambda_{0} A(t)\right\rangle / 2,
$$

where the symmetric logarithmic derivative is defined via $\left.2 \partial \varepsilon^{\prime} \rho_{\varepsilon}^{\prime}\right|_{\varepsilon^{\prime}=\varepsilon}=\left(\Lambda_{\varepsilon} \rho_{\varepsilon}+\rho_{\varepsilon} \Lambda_{\varepsilon}\right)$. This response function can now be rewritten as

$$
\begin{aligned}
\mathcal{R}_{\text {Mehboudi }}(t) & =-d_{t} \operatorname{Tr}\left\{\left(A(t) \Lambda_{0}+\Lambda_{0} A(t)\right) \pi_{0}\right\} / 2 \\
& =-d_{t} \operatorname{Tr}\left\{\left(\pi_{0} \Lambda_{0}+\Lambda_{0} \pi_{0}\right) A(t)\right\} / 2 \\
& =-d_{t} \operatorname{Tr}\left\{\left.\partial \varepsilon \pi_{\varepsilon}\right|_{\varepsilon=0} A(t)\right\} .
\end{aligned}
$$

Since $\pi_{\varepsilon}=\pi_{0}+\varepsilon \pi_{1}$ and $\left.\partial \varepsilon \pi_{\varepsilon}\right|_{\varepsilon=0}=\pi_{1}$, we finally have

$$
\mathcal{R}_{\text {Mehboudi }}(t)=\frac{d}{d t} \operatorname{Tr}\left\{A(t) \pi_{1}\right\}=\frac{d}{d t}\left\langle A(t) \pi_{1} \pi_{0}^{-1}\right\rangle,
$$

which is again equivalent to Eq. (3).

Response functions are intimately related to the Fisher information [35]. Two quantum generalizations of the Fisher information are commonly considered: the Kubo-MoriBogoliubov inner product (KMB) and the symmetric logarithmic derivative (SLD) [36,37]. Their main difference lies 
in the order of the noncommuting quantum observables. The SLD is most suited from the viewpoint of quantum estimation theory as it corresponds to the Cramer-Rao bound, while the KMB appears as the most natural quantum analog of the Fisher information from the viewpoint of statistical physics; it is indeed closely related to the canonical correlation of equilibrium linear response theory [29]. Equation (B3) can thus been regarded as a steady-state extension of the SLD approach, while Eq. (5) of the main text may be viewed as a steady-state generalization of the KMB approach.

\section{APPENDIX C: CALCULATIONS FOR THE COUPLED-OSCILLATOR MODEL}

We first determine the steady-state solution by introducing following Ref. [34] the symmetric characteristic function $\chi\left(\alpha_{1}, \alpha_{2}\right)=\left\langle D_{1}\left(\alpha_{1}\right) \otimes D_{2}\left(\alpha_{2}\right)\right\rangle$, where $D_{i}\left(\alpha_{i}\right)=\exp \left(\alpha_{i} a_{i}^{\dagger}-\right.$ $\left.\alpha_{i}^{*} a_{i}\right)$ is the displacement operator. The symmetric moments are directly obtained by differentiation,

$$
\left\langle a_{i}^{\dagger k} a_{j}^{l}\right\rangle_{s}=\left.\frac{d^{k}}{d \alpha_{i}^{k}} \frac{d^{l}}{\left(-\alpha_{j}^{*}\right)^{l}} \chi\left(\alpha_{1}, \alpha_{2}\right)\right|_{\alpha_{1}=\alpha_{2}=0},
$$

where $\langle\cdot\rangle_{s}$ is the expectation value of the symmetrized version of the operators $a_{i}^{\dagger k} a_{j}^{l}$. The equation for the characteristic function can be derived from the quantum Liouville equation by using

$$
\frac{d}{d t} \chi\left(\alpha_{1}, \alpha_{2}\right)=\operatorname{Tr}\left\{D_{1}\left(\alpha_{1}\right) \otimes D_{2}\left(\alpha_{2}\right) \dot{\rho}\right\},
$$

together with the identities,

$$
\begin{aligned}
& D_{i} a_{i}^{\dagger}=\left(-\frac{\alpha_{i}^{*}}{2}+\frac{d}{d \alpha_{i}}\right) D_{i}, D_{i} a_{i}=\left(-\frac{\alpha_{i}}{2}-\frac{d}{d \alpha_{i}^{*}}\right) D_{i}, \\
& a_{i}^{\dagger} D_{i}=\left(\frac{\alpha_{i}^{*}}{2}+\frac{d}{d \alpha_{i}}\right) D_{i}, a_{i} D_{i}=\left(\frac{\alpha_{i}}{2}-\frac{d}{d \alpha_{i}^{*}}\right) D_{i} .
\end{aligned}
$$

We then obtain the differential equation

$$
\begin{aligned}
\frac{d \chi}{d t}=\left\{\sum_{j=1}^{2}[\right. & \left.\omega_{j}\left(x_{j} \frac{d}{d p_{j}}-p_{j} \frac{d}{d x_{j}}\right)-\frac{\gamma_{j}}{2}\left(2 n_{j}+1\right)\left(x_{j}^{2}+p_{j}^{2}\right)-\frac{\gamma_{j}}{2}\left(x_{j} \frac{d}{d x_{j}}+p_{j} \frac{d}{d p_{j}}\right)\right] \\
& \left.+\lambda\left(x_{2} \frac{d}{d p_{1}}-p_{2} \frac{d}{d x_{1}}+x_{1} \frac{d}{d p_{2}}-p_{1} \frac{d}{d x_{2}}\right)\right\} \chi,
\end{aligned}
$$

with $x_{i}=\left(a_{i}^{\dagger}+a_{i}\right) / \sqrt{2}$ and $p_{i}=i\left(a_{i}-a_{i}^{\dagger}\right) / \sqrt{2}$, as well as $\alpha_{i}=x_{i}+i p_{i}$ and $d / d \alpha_{i}=\left(d / d x_{i}-i d / d p_{i}\right) / 2$. Equation $(\mathrm{C} 4)$ can be be solved by using the Gaussian ansatz $\chi\left(x_{1}, p_{1}, x_{2}, p_{2}\right)=\exp \left(i \vec{P} \overrightarrow{\bar{y}}-\vec{P}^{T} \bar{\sigma} \vec{P} / 2\right)$ with $\vec{P}=\left(x_{1}, p_{1}, x_{2}, p_{2}\right)^{T}$ and $\overrightarrow{\bar{y}}=$ $\left(\bar{y}_{1}, \bar{z}_{1}, \bar{y}_{2}, \bar{z}_{2}\right)^{T}$. Using Eq. $(\mathrm{C} 1)$, the variables $\left(\bar{y}_{i}, \bar{z}_{i}\right)$ and the matrix $\bar{\sigma}$ can be related to the average quadratures and the covariance matrix $\sigma$ via

$$
\begin{aligned}
\left\langle x_{i}\right\rangle & =\frac{\bar{z}_{i}}{\sqrt{2}}, \quad\left\langle p_{i}\right\rangle=-\frac{\bar{y}_{i}}{\sqrt{2}}, \\
\sigma_{x_{i} x_{j}} & =\frac{1}{2} \bar{\sigma}_{p_{i} p_{j}}, \quad \sigma_{p_{i} p_{j}}=\frac{1}{2} \bar{\sigma}_{x_{i} x_{j}}, \quad \sigma_{x_{i} p_{j}}=-\frac{1}{2} \bar{\sigma}_{x_{j} p_{i}}
\end{aligned}
$$

The steady-state solution is given by vanishing first moments, $\left\langle x_{1,2}\right\rangle=\left\langle p_{1,2}\right\rangle=0$, and the covariance matrix,

$$
\sigma=\zeta\left(\begin{array}{cccc}
D+n_{1}+\frac{1}{2} & 0 & -\delta C & -\gamma C \\
0 & D+n_{1}+\frac{1}{2} & \gamma C & -\delta C \\
-\delta C & \gamma C & D+n_{2}+\frac{1}{2} & 0 \\
-\gamma C & -\delta C & 0 & D+n_{2}+\frac{1}{2}
\end{array}\right),
$$

with the three parameters

$$
\begin{aligned}
\zeta & =\frac{\gamma^{2}+\delta^{2}}{4 \lambda^{2}+\gamma^{2}+\delta^{2}}, \\
D & =\frac{2 \lambda^{2}\left(n_{1}+n_{2}+1\right)}{\gamma^{2}+\delta^{2}}, \\
C & =\frac{\lambda\left(n_{1}-n_{2}\right)}{\gamma^{2}+\delta^{2}}
\end{aligned}
$$

The time evolution of the observable of interest $A$ is obtained by solving the adjoint master equation by finding operators that form a closed set of linear differential equations [26]. For the considered quadratic system, these operators are given by $\vec{v}(t)^{T}=$ $\left(a_{1}^{\dagger} a_{1}(t), a_{1}^{2}(t), a_{1}^{\dagger 2}(t), a_{2}^{\dagger} a_{2}(t), a_{2}^{2}(t), a_{2}^{\dagger 2}(t), a_{1} a_{2}(t), a_{1} a_{2}^{\dagger}(t), a_{1}^{\dagger} a_{2}(t), a_{1}^{\dagger} a_{2}^{\dagger}(t)\right)$. They obey the ensemble of linear differential equations

$$
\frac{d}{d t} \vec{v}(t)=M \vec{v}(t)+\vec{w},
$$




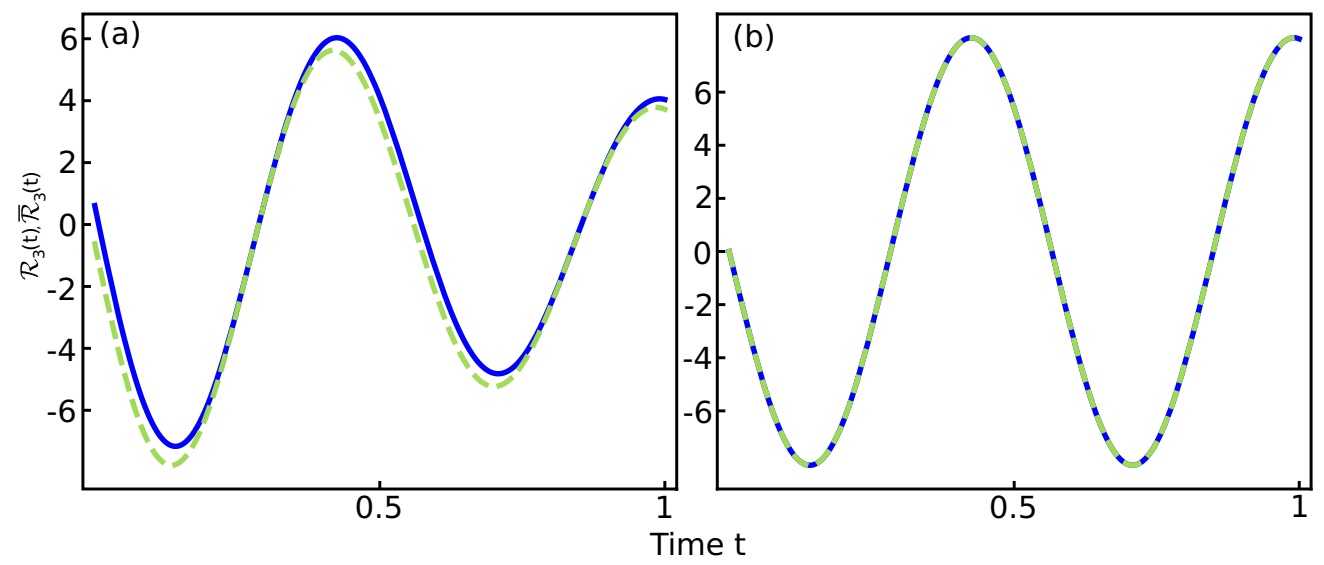

FIG. 4. Violation of detailed balance in the two-oscillator model. (a) The steady-state response $\mathcal{R}_{3}(\tau)$, Eq. (D1), (green dashed) is not equal to $\overline{\mathcal{R}}_{3}(t)$, Eq. (D2) (blue solid), especially at short times, as required by the detailed balance condition. (b) By contrast, detailed balance is obeyed, $\mathcal{R}_{3}(\tau)=\overline{\mathcal{R}}_{3}(t)$, in the unitary limit $\gamma \rightarrow 0$.

with $\vec{w}=\left(n_{1} \gamma, 0,0, n_{2} \gamma, 0,0,0,0,0,0\right)$. The matrix $M$ explicitly reads

$M=\left(\begin{array}{cccccccccc}-\gamma & 0 & 0 & 0 & 0 & 0 & 0 & i \lambda & -i \lambda & 0 \\ 0 & -2 i \omega_{1}-\gamma & 0 & 0 & 0 & 0 & -2 i \lambda & 0 & 0 & 0 \\ 0 & 0 & 2 i \omega_{1}-\gamma & 0 & 0 & 0 & 0 & 0 & 0 & 2 i \lambda \\ 0 & 0 & 0 & -\gamma & 0 & 0 & 0 & -i \lambda & i \lambda & 0 \\ 0 & 0 & 0 & 0 & -2 i \omega_{2}-\gamma & 0 & -2 i \lambda & 0 & 0 & 0 \\ 0 & 0 & 0 & 0 & 0 & 2 i \omega_{2}-\gamma & 0 & 0 & 0 & 2 i \lambda \\ 0 & -i \lambda & 0 & 0 & -i \lambda & 0 & -i \omega_{12}-\gamma & 0 & 0 & 0 \\ i \lambda & 0 & 0 & -i \lambda & 0 & 0 & 0 & -i \Delta \omega-\gamma & 0 & 0 \\ -i \lambda & 0 & 0 & i \lambda & 0 & 0 & 0 & 0 & i \Delta \omega-\gamma & 0 \\ 0 & 0 & i \lambda & 0 & 0 & i \lambda & 0 & 0 & 0 & i \omega_{12}-\gamma\end{array}\right)$

(C12)

with $\omega_{12}=\omega_{1}+\omega_{2}$ and $\Delta \omega=\omega_{1}-\omega_{2}$. The time dependence of the number operator, $a_{1}^{\dagger} a_{1}$, of the first oscillator follows via matrix exponentiation,

$$
\begin{aligned}
a_{1}^{\dagger} a_{1}(t)= & f(t) a_{1}^{\dagger} a_{1}+g(t) a_{1}^{2}+h(t) a_{1}^{\dagger 2}+j(t) a_{2}^{\dagger} a_{2} \\
& +l(t) a_{2}^{2}+m(t) a_{2}^{\dagger 2}+n(t) a_{1} a_{2}+p(t) a_{1} a_{2}^{\dagger} \\
& +q(t) a_{1}^{\dagger} a_{2}+r(t) a_{1}^{\dagger} a_{2}^{\dagger}+s(t) .
\end{aligned}
$$

The various functions appearing in Eq. (C13) are given by

$$
\begin{aligned}
f(t) & =e^{-\gamma t}\left(\delta^{2}+2 \lambda^{2}+2 \lambda^{2} \cos z t\right) / z^{2}, \\
j(t) & =-2 \lambda^{2} e^{-\gamma t}(\cos z t-1) / z^{2}, \\
p(t) & =\lambda e^{-\gamma t}(-\delta+i z \sin z t+\delta \cos z t) / z^{2}, \\
q(t) & =\lambda e^{-\gamma t}(-\delta-i z \sin z t+\delta \cos z t) / z^{2}, \\
r(t) & =0, \quad g(t)=0, \quad h(t)=0, \\
l(t) & =0, \quad m(t)=0, \quad n(t)=0,
\end{aligned}
$$

together with

$$
\begin{aligned}
s(t)= & \frac{1}{z^{3}\left(\gamma^{2}+\delta^{2}+4 \lambda^{2}\right)} e^{-\gamma t} \\
& \times\left\{z \left[\left(\gamma^{2}+z^{2}\right)\left(\delta^{2} n_{1}+2 \lambda^{2}\left(n_{1}+n_{2}\right)\right)\right.\right. \\
& \left.-z^{2} e^{\gamma t}\left(n_{1}\left(\gamma^{2}+\delta^{2}\right)+2 \lambda^{2}\left(n_{1}+n_{2}\right)\right)\right] \\
& \left.+2 \gamma \lambda^{2}\left(n_{1}-n_{2}\right)\left(\gamma z \cos z t-z^{2} \sin z t\right)\right\} .
\end{aligned}
$$

For $\lambda=0$, Eq. (C13) simplifies to $a_{1}^{\dagger} a_{1}(t)=e^{-\gamma t} a_{1}^{\dagger} a_{1}+(1-$ $\left.e^{-\gamma t}\right) n_{1}$, as expected for a thermal oscillator [26].

\section{APPENDIX D: VIOLATION OF DETAILED BALANCE IN THE TWO-OSCILLATOR MODEL}

We further show that the steady-state response function of the two-oscillator model does not satisfy the detailed balance condition $\langle A B(t)\rangle=\langle B A(t)\rangle$ [27]. Figure 4 exhibits the two response functions,

$$
\begin{gathered}
\mathcal{R}_{3}(\tau)=i \beta_{1} \hbar \omega_{1}\left\langle\left[a_{1}^{\dagger} a_{2}+a_{1} a_{2}^{\dagger}, a_{1}^{\dagger} a_{1}(\tau)\right]\right\rangle, \\
\overline{\mathcal{R}}_{3}(\tau)=i \beta_{1} \hbar \omega_{1}\left\langle\left[a_{1}^{\dagger} a_{1},\left(a_{1}^{\dagger} a_{2}+a_{1} a_{2}^{\dagger}\right)(\tau)\right]\right\rangle .
\end{gathered}
$$

We observe that detailed balance is violated, $\mathcal{R}_{3}(\tau) \neq \overline{\mathcal{R}}_{3}(\tau)$, especially at short times, where an offset between the two curves is visible. This violation disappears in the unitary limit $\gamma \rightarrow 0$.

\section{APPENDIX E: RESPONSE FOR THE TWO-OSCILLATOR MODEL FOR $\delta=0$}

We here explicitly show that the steady-state response may be different from zero in cases where the equilibrium response vanishes. For zero detuning, $\delta=0$, the perturbation commutes with the unperturbed Hamiltonian, $\left[H_{0}, H_{I}\right]=$ $\hbar \omega_{1}\left[a_{1}^{\dagger} a_{1}+a_{2}^{\dagger} a_{2}+\lambda H_{I}, a_{1}^{\dagger} a_{2}+a_{1} a_{2}^{\dagger}\right]=0$, implying that the 
response vanishes for a thermal state. However, in that limit the response function (11) of the main text reads

$$
\mathcal{R}_{\delta=0}(\tau)=e^{-\gamma \tau} \Delta n \gamma \frac{2 \lambda \cos (2 \lambda \tau)+\gamma \sin (2 \lambda \tau)}{\left(\gamma^{2}+4 \lambda^{2}\right)\left(\beta_{1} \hbar \omega_{1}\right)^{-1}},
$$

which is in general nonzero. The thermal response thus vanishes for $\lambda \neq 0$, while the steady-state response is finite. In the thermal limit, $\lambda \rightarrow 0$, Eq. (E1) clearly vanishes, as it should.
[1] H. Nyquist, Thermal agitation of electric charge in conductors, Phys. Rev. 32, 110 (1928).

[2] H. B. Callen and T. A. Welton, Irreversibility and generalized noise, Phys. Rev. 83, 34 (1951).

[3] R. Kubo, Statistical-mechanical theory of irreversible Processes. I. General theory and simple applications to magnetic and conduction problems, J. Phys. Soc. Jpn. 12, 570 (1957).

[4] R. Kubo, The fluctuation-dissipation theorem, Rep. Prog. Phys. 29, 255 (1966).

[5] P. Hänggi and H. Thomas, Stochastic processes: time-evolution, symmetries and linear response, Phys. Rep. 88, 207 (1982).

[6] R. Zwanzig, Nonequilibrium Statistical Mechanics (Oxford University Press, Oxford, 2001).

[7] R. Kubo, M. Toda, and N. Hatshitsume, Statistical Physics II (Springer, Berlin, 1978).

[8] U. M. B. Marconi, A. Puglisi, L. Rondoni, and A. Vulpiani, Fluctuation-dissipation: Response theory in statistical physics, Phys. Rep. 461, 111 (2008).

[9] L. F. Cugliandolo, D. S. Dean, and J. Kurchan, FluctuationDissipation Theorems and Entropy Production in Relaxational Systems, Phys. Rev. Lett. 79, 2168 (1997).

[10] G. S. Agarwal, Fluctuation-dissipation theorems for systems in non-thermal equilibrium and applications, Z. Phys. 252, 25 (1972).

[11] L. F. Cugliandolo, J. Kurchan, and G. Parisi, Off equilibrium dynamics and aging in unfrustrated systems, J. Phys. I France 4, 1641 (1994).

[12] T. Speck and U. Seifert, Restoring a fluctuation-dissipation theorem in a nonequilibrium steady state, EPL 74, 391 (2006).

[13] R. Chetrite, G. Falkovich, and K. Gawedzki, Fluctuation relations in simple examples of non-equilibrium steady states, J. Stat. Mech. (2008) P08005.

[14] J. Prost, J. Joanny, and J. M. R. Parrondo, Generalized Fluctuation-Dissipation Theorem for Steady State Systems, Phys. Rev. Lett. 103, 090601 (2009).

[15] M. Baiesi, C. Maes, and B. Wynants, Fluctuations and Response of Nonequilibrium States, Phys. Rev. Lett. 103, 010602 (2009).

[16] V. Blickle, T. Speck, C. Lutz, U. Seifert, and C. Bechinger, Einstein relation generalized to nonequilibrium, Phys. Rev. Lett. 98, 210601 (2007).

[17] J. R. Gomez-Solano, A. Petrosyan, and S. Ciliberto, Experimental Verification of a Modified Fluctuation-Dissipation Relation for a Micron-Sized Particle in a Nonequilibrium Steady State, Phys. Rev. Lett. 106, 200602 (2009).

[18] J. Mehl, V. Blickle, U. Seifert, and C. Bechinger, Experimental accessibility of generalized fluctuation-dissipation relations for nonequilibrium steady states, Phys. Rev. E 82, 032401 (2010).

[19] S. Ciliberto, R. Gomez-Solano, and A. Petrosyan, Fluctuations, linear response, and currents in out-of-equilibrium systems, Annu. Rev. Condens. Matter Phys. 4, 235 (2013).

[20] U. Seifert and T. Speck, Fluctuation-dissipation theorem in nonequilibrium steady states, Europhys. Lett. 89, 10007 (2010).

[21] M. Baiesi and C. Maes, An update on the nonequilibrium linear response, New J. Phys. 15, 013004 (2013).

[22] W. Weidlich, Fluctuation-dissipation theorem for a class of stationary open systems, Z. Phys. 248, 234 (1971).

[23] R. Chetrite and K. Mallick, Quantum fluctuation relations for the Lindblad master equation, J. Stat. Phys. 148, 480 (2012).

[24] M. Mehboudi, A. Sanpera, and J. M. R. Parrondo, Generalized fluctuation-dissipation relation for quantum Markovian systems, Quantum 2, 66 (2018).

[25] L. C. Venuti and P. Zanardi, Dynamical response theory for driven-dissipative quantum systems, Phys. Rev. A 93, 032101 (2016).

[26] H. P. Breuer and F. Petruccione, The Theory of Open Quantum Systems (Oxford University Press, Oxford, 2002).

[27] G. Agarwal, Open quantum markovian systems and the microreversibility, Z. Physik 258, 409 (1973).

[28] S. Amari and H. Nagaoka, Methods of Information Geometry (Oxford University Press, Oxford, 2000), Eq. (7.61).

[29] D. Petz and G. Toth, The Bogoliubov inner product in quantum statistics, Lett. Math. Phys. 27, 205 (1993).

[30] H. Carmichael, An Open Systems Approach to Quantum Optics (Springer, Berlin, 1993).

[31] M. Aspelmeyer, T. J. Kippenberg, and F. Marquardt, Cavity optomechanics, Rev. Mod. Phys. 861391 (2014).

[32] J. Javanainen, Oscillatory exchange of atoms between traps containing Bose condensates, Phys. Rev. Lett. 57, 3164 (1986).

[33] T. Brandes, Coherent and collective quantum optical effects in mesoscopic systems, Phys. Rep. 408, 315 (2005).

[34] S. Campbell, G. De Chiara, and M. Paternostro, Equilibration and nonclassicality of a double-well potential, Sci. Rep. 6, 19730 (2016).

[35] T. Shitara and M. Ueda, Determining the continuous family of quantum Fisher information from linear response theory, Phys. Rev. A 94, 062316 (2016).

[36] M. Hayashi, Two quantum analogues of Fisher information from a large deviation viewpoint of quantum estimation, J. Phys. A: Math. Gen. 35, 7689 (2002)

[37] M. Hayashi, Quantum Information (Springer, Berlin, 2006). 